\title{
Capillary pressure may predict preclinical changes in the eye
}

\author{
K. M. Gooding $\cdot$ J. E. Tooke $\cdot$ H. von Lany $\cdot$ M. Mitra . \\ R. Ling • C. I. Ball • D. Mawson • K. Skinner • \\ A. C. Shore
}

Received: 29 October 2009 / Accepted: 30 April 2010 /Published online: 6 June 2010

(C) The Author(s) 2010. This article is published with open access at Springerlink.com

\begin{abstract}
Aims/hypothesis Microvascular dysfunction is associated with end-organ damage. Macular oedema is an important component of diabetic retinopathy. Macular thickness can be accurately quantified by optical coherence tomography (OCT), enabling accurate assessment of the macular prior to clinically apparent abnormalities. We investigated whether macular (fovea) thickness in non-diabetic individuals is related to the microvascular variables controlling fluid filtration across a blood vessel wall, in particular capillary pressure and the microvascular filtration capacity (Kf). Methods We recruited 50 non-diabetic individuals $(25 \mathrm{men}$, 25 women; age range: $26-78$ years; BMI range: $20-46 \mathrm{~kg} / \mathrm{m}^{2}$ ). Fovea thickness was assessed by OCT. Microvascular assessments included: finger nailfold capillary pressure; Kf; microvascular structural assessments, i.e. skin vasodilatory capacity, minimum vascular resistance (MVR) and microvascular distensibility; and endothelial function.
\end{abstract}

Electronic supplementary material The online version of this article (doi:10.1007/s00125-010-1805-x) contains supplementary material, which is available to authorised users.

K. M. Gooding $(\bowtie) \cdot$ J. E. Tooke $\cdot$ C. I. Ball $\cdot$ D. Mawson $\cdot$

K. Skinner $\cdot$ A. C. Shore

Diabetes and Vascular Medicine,

Institute of Biomedical and Clinical Science,

Peninsula Medical School,

Barrack Road,

Exeter EX2 5AX, UK

e-mail: kim.gooding@pms.ac.uk

K. M. Gooding • C. I. Ball • D. Mawson - K. Skinner · A. C. Shore

Peninsula National Institute of Health Research

Clinical Research Facility,

Exeter, UK

H. von Lany $\cdot$ M. Mitra $\cdot$ R. Ling

West of England Eye Unit,

Royal Devon and Exeter NHS Foundation Trust,

Exeter, UK
Results At 214.6 (19.9) $\mu \mathrm{m}$ (mean [SD]), fovea thickness was within normal range. Capillary pressure, adjusted for BMI, was associated with fovea thickness (standardised beta $0.573, p=0.006$, linear regression). Fovea thickness was not associated with $\mathrm{Kf}$, microvascular structural assessments or endothelial function. Capillary pressure was still associated with fovea thickness when adjusted for microvascular variables (Kf, vasodilatory capacity, MVR, microvascular distensibility or endothelial function), or for risk factors for diabetes (systemic blood pressure, insulin sensitivity, inflammation, glycaemic status and lipids) and age.

Conclusions/interpretation Capillary pressure, a key determinant of movement of fluid across a blood vessel wall, is associated with fovea thickness in non-diabetic individuals. This suggests that with regard to potential preventative or therapeutic targets, attention should be directed at the mechanisms determining retinal microvascular pressure.

Keywords Capillary pressure - Fluid filtration .

Fovea thickness $\cdot$ Macular oedema $\cdot$ Obesity

$\begin{array}{ll}\text { Abbreviations } \\ \text { CSMO } & \text { Clinically significant macular oedema } \\ \text { ITT } & \text { Insulin tolerance test } \\ \text { Kf } & \text { Microvascular filtration capacity } \\ \text { LDPI } & \text { Laser Doppler perfusion imager } \\ \text { MVR } & \text { Minimum vascular resistance } \\ \text { OCT } & \text { Optical coherence tomography }\end{array}$

\section{Introduction}

The microcirculation is the site where nutrients and waste products are exchanged between the circulation and tissues. Normal microcirculation is vital for tissue health, while abnormalities are associated with disease. In the Athero- 
sclerosis Risk in Communities and other epidemiological studies, clinically apparent retinal structural microvascular abnormalities (including non-diabetic retinopathy) were, for example, independently related to risk of diabetes [1-5], impaired fasting hyperglycaemia $[1,6]$, stroke [7-11], magnetic resonance imaging-defined subclinical cerebral infarcts [12], progression of cerebral small vessel disease [1], congestive heart failure [4], coronary heart disease and acute myocardial infarction $[5,8,11]$, left ventricular hypertrophy [13] and cardiovascular mortality rates [14].

However, it is not only these clinically apparent structural abnormalities in the retinal microcirculation that are related to clinical outcomes and/or risk. In the periphery, functional impairments in the small vessels, such as impaired endothelial function of the forearm resistance blood vessels or impaired skin microcirculatory responsiveness, are associated with cardiovascular risk factors $[15,16]$ and end-organ damage (intraventricular septal thickness or urinary albumin excretion) [17].

Indeed, graded abnormalities in the reactive hyperaemic response of the skin occur across levels of urinary albumin excretion rate from 2 to $20 \mu \mathrm{g} / \mathrm{min}$, i.e. within the normal range [18]. These associations indicate that subtle abnormalities of microvascular function may run parallel to early evidence of end-organ damage even prior to presentation of clinical disorders.

Diabetic retinopathy remains a leading cause of blindness with maculopathy being the most resistant to amelioration. Macular (fovea) thickening is a preclinical sign of macular oedema, which can be quantified using the new, revolutionary technique of retinal optical coherence tomography (OCT).

This study investigated the mechanisms underlying preclinical changes in fovea thickness, and in particular the roles of the variables that can control fluid movement across the blood vessel wall, e.g. capillary pressure and microvascular filtration capacity (Kf).

\section{Methods}

Participants We recruited 50 non-diabetic individuals (age range 26 to 78 years) from the local community by advertisements for this study (participant characteristics, see Table 1). Exclusion criteria were diabetes, overt cardiovascular disease, Raynaud's phenomenon, age less than 18 or over 80 years, and use of any anti-hypertensive, oral hypoglycaemic or lipid-lowering therapies. Seven of the participants had the metabolic syndrome, as defined by the International Diabetes Federation [19]. None of the participants had clinically defined microalbuminuria. Only one participant smoked (cigars), but this participant refrained from smoking on the mornings of the study.
Table 1 Participant characteristics

\begin{tabular}{lll}
\hline Variables & Mean (SD) & Range \\
\hline Total sample $(n)$ & $50(25)$ & \\
Men $(n)$ & 25 & \\
Age $($ years $)$ & $53.5(11.8)$ & 26 to 78 \\
BMI $\left(\mathrm{kg} / \mathrm{m}^{2}\right)$ & $28.6(6.1)$ & 20 to 46 \\
Waist circumference (cm) & $94.4(15.8)$ & 69 to 139 \\
Systolic blood pressure (mmHg) & $124(16)$ & 87 to 162 \\
Diastolic blood pressure (mmHg) & $75.2(9.4)$ & 59 to 95 \\
Fasting glucose (mmol/l) & $4.9(0.5)$ & 4.0 to 6.9 \\
HbA $(\%)$ & $5.5(0.4)$ & 3.9 to 6.3 \\
Total cholesterol (mmol/l) & $5.37(0.84)$ & 3.7 to 7.4 \\
HDL-cholesterol (mmol/l) & $1.54(0.42)$ & 0.72 to 2.32 \\
LDL-cholesterol (mmol/l) & $3.21(0.62)$ & 2.17 to 5.22 \\
Triacylglycerol (mmol/l) & $1.19(0.46)$ & 0.46 to 2.40 \\
ACR (mg/mmol) & $0.62(0.27)$ & 0.22 to 1.44 \\
AER( $\mu$ g/min) & $5.35(2.82)$ & 2.11 to 15.36 \\
Inflammation markers & & \\
C-reactive protein (ng/ml) & $982(1,293)$ & 24 to 5,578 \\
Measures of insulin sensitivity & & \\
ITT (mmol ${ }^{-1}$ min $\left.{ }^{-1}\right)$ & $-0.22(0.08)$ & -0.53 to -0.11 \\
Insulin sensitivity $(\mathrm{HOMA})(\%)$ & $130(74)$ & 16 to 318 \\
\hline
\end{tabular}

ACR, albumin:creatinine ratio

Ethics approval was granted by the Local Exeter and North Devon Medical Research Ethics Committee, and written informed consent was obtained from all participants. The studies conformed to the principles outlined in the Declaration of Helsinki.

Procedures Participants attended the study visits in the morning following an overnight fast. The following assessments were spread over two to three mornings: macular thickness, retinal photography, capillary pressure, Kf, microvascular structural assessments (vasodilatory capacity, minimum vascular resistance [MVR], microvascular distensibility), resting skin perfusion, microvascular endothelial function and supine blood pressure (mean of three measurements taken at $1 \mathrm{~min}$ intervals). The vascular assessments were performed in temperature-controlled microvascular laboratories with the participants in a supine position following a $30 \mathrm{~min}$ acclimatisation period.

Macular thickness OCT was used to quantify retinal thickness (Stratus OCT 3000; Carl Zeiss, Welwyn Garden City, UK) using the fast macular thickness scanning protocol following pupil dilation in both eyes. OCT measures the macular thickness between the vitreoretinal interface and the interface between the inner and outer photoreceptors segments by changes in reflectivity from the 
reflected light (original light source: broad bandwidth near infrared light beam with central wavelength of $820 \mathrm{~nm}$ ). The Stratus OCT software (version 4.0.7) automatically determines the thickness of the subdivisions of the macula (fovea; inner and outer quadrants of the superior, nasal, inferior and temporal regions) and the volume of the total macular area. The fovea was defined as the central disc of the scan, with a radius of $500 \mu \mathrm{m}$. This technique is reproducible in healthy participants [20]. In our hands, the intra-individual coefficient of variation for the assessment of fovea thickness was $2.6 \%(182.0$ [4.8] $\mu \mathrm{m}$, mean [SD]) determined from one participant on four separate occasions.

Capillary pressure Finger nailfold capillary pressure was measured following direct cannulation of the apex of the capillary loop with a glass micropipette. Dynamic recordings of capillary pressure were achieved using an electronic resistance feedback servo-nulling system [21]. Day-to-day intra-participant variation of the measurement of capillary pressure was $5.2 \%(3.6 \%)$ in six individuals studied on three separate occasions.

Microvascular filtration capacity $\mathrm{Kf}$ was determined using strain-gauge plethysmography (Filtrass 2001; Domed Medizintechnik, Munich, Germany) [22]. The system uses an occlusion cuff placed proximal (thigh) to the filtrass sensor to monitor changes in calf circumference and hence volume in response to increases in venous congestion pressure. The calf circumference is continuously monitored throughout a series of small pressure increases of $8 \mathrm{mmHg}$ that in total do not exceed diastolic pressure. As the venous congestion pressure increases, the net movement of fluid across the vessel wall into the interstitium, that is fluid filtration, increases. The relationship between fluid filtration and cuff pressure is linear, with the slope representing $\mathrm{Kf}$. The mean day-to-day intra-participant variation in the measurement of $\mathrm{Kf}$ using this system was $7.1 \%$ in two healthy participants assessed on four separate occasions $\left(2.37 \pm 0.21 \times 10^{-3}\right.$ and $2.90 \pm 0.15 \times 10^{-3} \mathrm{ml} \mathrm{min}^{-1}\left[100 \mathrm{ml}^{-1} \mathrm{mmHg}^{-1}\right.$, mean $\pm \mathrm{SD}$, for participants 1 and 2 respectively).

Structural microvascular assessments: vasodilatory capacity, minimum vascular resistance and microvascular distensibility Maximum hyperaemia was assessed by heating a small area of skin to 42 to $44^{\circ} \mathrm{C}$ [23], which induces maximum hyperaemia and reflects the vasodilatory capacity of the skin microcirculation. This was achieved by attaching a small brass heater (area $0.76 \mathrm{~cm}^{2}$ ) (Moor Instruments, Axminster, UK) to the dorsum of the foot for $30 \mathrm{~min}$. The resultant maximum hyperaemic response was assessed by single point laser Doppler fluximetry (Perflux Pf2: Perimed, Jarfalla, Sweden), with eight equally spaced measurements being made within the heated area and the mean used to represent vasodilatory capacity, arbitrarily expressed as volts. The foot was then lowered by $50 \mathrm{~cm}$ and blood flow reassessed in the eight sites in the dependent position. Microvascular distensibility was calculated by dividing dependent vasodilatory capacity by horizontal vasodilatory capacity. The mean day to day intra-participant coefficient of variation for vasodilatory capacity in three participants assessed on five separate occasions was $6.6 \%$; in a male participant assessed over a 2 year period using laser Doppler fluximetry, it was $7.1 \%(2.11$ [0.15] arbitrary units, mean $[\mathrm{SD}])$. MVR was calculated by dividing the mean arterial blood pressure by vasodilatory capacity.

Endothelial function Endothelial function was assessed by iontophoretic application of the endothelial-dependent vasodilator acetylcholine [24]. Iontophoresis delivers the drug of interest to the skin using a small electric charge. Briefly, a perspex chamber, with an internal area of $0.76 \mathrm{~cm}^{2}$ available for iontophoresis, was attached to the volar aspect of the forearm and an indifferent electrode (hydro gel pad) attached to the wrist. An electric charge was applied using a battery powered iontophoresis controller (MIC-1; Moor instruments, Axminster, UK) to transfer the study drug across the skin. To assess the microvascular effect of acetylcholine, 1\% acetylcholine (Miochol-E; Novartis Pharmaceuticals, Horsham, UK) was iontophoresed at one site and its vehicle, mannitol (3\%) (Royal Devon and Exeter Hospital Pharmacy, Exeter, UK), was iontophoresed at another site. The protocol involved the application of a positive charge for five $20 \mathrm{~s}$ pulses of $100 \mu \mathrm{A}$, with a $60 \mathrm{~s}$ rest period between each pulse, making a total charge of $10 \mathrm{mC}$. The skin hyperaemic response was quantified by a laser Doppler perfusion imager (LDPI) (LDPI Pim 2.0; Perimed, Jarfalla, Sweden). Skin hyperaemia was assessed at baseline and then every $40 \mathrm{~s}$ for $400 \mathrm{~s}$ after the start of the charge. The LDPI perfusion images were saved for later off-line analysis. For each image, the mean perfusion over the entire iontophoresed area $\left(0.76 \mathrm{~cm}^{2}\right)$ was calculated with LDPI Win 2.0.9 software (Perimed) and arbitrarily expressed as volts. The results are expressed as the peak hyperaemic response. Resting skin perfusion was determined from baseline scan taken at the mannitol (acetylcholine vehicle) site.

Insulin sensitivity assessments Central insulin sensitivity was calculated by HOMA of insulin sensitivity, based on fasting insulin and glucose levels [25]. Peripheral insulin sensitivity was assessed using a 15 min continuous insulin tolerance test (ITT). A bolus of $0.1 \mathrm{U} / \mathrm{kg}$ insulin (Human Actrapid; Novo Nordisk, Crawley, UK) was administered into a vein at the site of the antecubital fossa. Blood samples for determination of blood glucose were taken each minute until the blood glucose level reached $3 \mathrm{mmol} / \mathrm{l}$ or 
15 min duration was reached. The glucose samples were measured in duplicate (YSI2300 Stat Plus; YSI Life Sciences, Yellowsprings, OH, USA). The slope of the blood glucose curve was used to represent insulin sensitivity. Mean day-to-day intra-participant variation of the measurement of insulin sensitivity by ITT was $18 \%$ in four individuals studied on three separate occasions.

Haemostatic and urine assessments Standard haemostatic and urine measurements included: $\mathrm{HbA}_{1 \mathrm{c}}$ (normal range 4.0$6.0 \%$ ); fasting glucose; lipid profile (total cholesterol, HDLcholesterol, LDL-cholesterol and triacylglycerol); urinary creatinine; and urinary albumin. A urine albumin excretion rate of 20 to $200 \mu \mathrm{g} / \mathrm{min}$ or an albumin:creatinine ratio $\geq 2.5 \mathrm{mg} / \mathrm{mmol}$ (men) or $\geq 3.5 \mathrm{mg} / \mathrm{mmol}$ (women) was regarded as clinical cut-off for microalbuminuria. Additional assessments included fasting plasma insulin $(0.05 \%$ crossreactivity with human proinsulin) (Cobas; Roche, Burgess Hill, UK) and C-reactive protein (Vascular injury panel II plate; Meso Scale Discovery, Gaithersburg, MD, USA).

Statistical analysis Data are presented as mean (SD) unless stated otherwise. Fovea thickness was our primary outcome measure, as it is the only region of the macular that is independently associated with macular oedema [26]. As there was no difference in fovea thickness or intraocular pressure between right and left eye, data from both eyes were averaged and used in subsequent data analysis. Univariate analysis (Pearson's or Spearman's correlation test, dependent on normality of data) was used to explore whether fovea thickness was associated with the following microvascular variables: capillary pressure, $\mathrm{Kf}$, vasodilatory capacity, MVR, microvascular distensibility, resting skin perfusion and endothelial function. Significant associations from univariate analysis were then further explored using linear regression, adjusting for BMI as it is well known that obesity influences measures of microvascular variables [27, 28] (K. M. Gooding, J. E. Tooke and A. C. Shore, unpublished observations). With a sample size of 50, the study was powered to detect, with up to three predictors in a model, a large effect size $\left(f^{2}=0.33\right)$ at the $5 \%$ level with $90 \%$ power.

\section{Results}

Fovea thickness was within the normal range defined by Chan et al [29] using the OCT 3000 system in all participants. There was no evidence of diabetes-related retinal abnormalities or ocular neurodegenerative disorders in any of the participants. Results are presented in Table 2.

Relationships between fovea thickness and microvascular variables Capillary pressure was significantly associated with fovea thickness $(r=0.379, p=0.027$, Model 1) (Table 3). This relationship was strengthened when the model was adjusted for BMI (Model 2) (Fig. 1). The relationship between capillary pressure and fovea thickness adjusted for BMI was not explained by (1) Kf (Model 3); (2) structural effects (vasodilatory capacity, MVR, microvascular distensibility) (capillary pressure standardised beta $\geq 0.547, p \leq 0.009$, Models 6-8) (Electronic supplementary material [ESM] Table 1); (3) resting skin perfusion (capillary pressure standardised beta $0.599, p=0.006$, Model 9) (ESM Table 1); or (4) endothelial function (standardised beta 0.562 , $p=0.008$, Model 10) (ESM Table 1). Neither Kf nor any of the other microvascular variables were independently associated with fovea thickness. In addition, no significant associations were observed between any of the microvascular variables and the inner and outer quadrants of the macular.

Explaining the relationship between fovea thickness and capillary pressure The relationship between capillary pressure adjusted for BMI and fovea thickness was not explained by mean arterial pressure (Model 4), systolic or diastolic blood pressure (results not shown) or by intraocular pressure (Model 5). In addition, the relationship between capillary pressure adjusted for BMI and fovea thickness was not explained by age (capillary pressure
Table 2 Results from eye and vascular assessments for the entire group

\begin{tabular}{lcc}
\hline Variable & Mean (SD) & Range \\
\hline Fovea thickness $(\mu \mathrm{m})$ & $214.6(19.9)$ & $171-249$ \\
Intraocular pressure $(\mathrm{mmHg})$ & $15.1(3.2)$ & $10-22$ \\
Capillary pressure $(\mathrm{mmHg})$ & $16.6(3.8)$ & $11.3-26.3$ \\
$\mathrm{Kf}\left(10^{-3} \mathrm{ml} \mathrm{min}^{-1}\left[100 \mathrm{ml}^{-1} \mathrm{mmHg}^{-1}\right)\right.$ & $3.58(0.97)$ & $1.00-6.00$ \\
$\mathrm{MVR}(\mathrm{mmHg} / \mathrm{V})$ & $52.6(21.3)$ & $26.0-141$ \\
Vasodilatory capacity $(\mathrm{V})$ & $1.93(0.64)$ & $0.56-3.21$ \\
Microvascular distensibility & $1.23(0.21)$ & $0.88-1.69$ \\
Resting skin perfusion $(\mathrm{V})$ & $0.18(0.07)$ & $0.01-0.29$ \\
Peak endothelial-dependent skin perfusion $(\mathrm{V})$ & $1.06(0.27)$ & $0.61-1.86$ \\
\hline
\end{tabular}


Table 3 Associations between fovea thickness and variables as indicated

\begin{tabular}{|c|c|c|c|c|c|}
\hline Associations per model & $R^{2}$ & $\begin{array}{l}p \text { value for overall } \\
\text { model }\end{array}$ & $\begin{array}{l}\text { Unstandardised beta } \\
\text { (SE) }\end{array}$ & $\begin{array}{l}\text { Standardised } \\
\text { beta }\end{array}$ & $\begin{array}{l}p \text { values for individual } \\
\text { predictors }\end{array}$ \\
\hline Model 1 & 0.140 & 0.027 & & & \\
\hline Capillary pressure & & & $2.067(0.892)$ & 0.374 & 0.027 \\
\hline Model 2 & 0.215 & 0.021 & & & \\
\hline Capillary pressure & & & $3.166(1.07)$ & 0.573 & 0.006 \\
\hline BMI & & & $-1.02(0.585)$ & -0.338 & 0.091 \\
\hline Model 3 & 0.188 & 0.115 & & & \\
\hline Capillary pressure & & & $2.897(1.222)$ & 0.501 & 0.025 \\
\hline $\mathrm{Kf}$ & & & $-1.119(4.799)$ & -0.048 & 0.121 \\
\hline BMI & & & $-1.042(0.652)$ & -0.647 & 0.817 \\
\hline Model 4 & 0.280 & 0.018 & & & \\
\hline Capillary pressure & & & $3.470(1.050)$ & 0.633 & 0.002 \\
\hline BMI & & & $-1.239(0.598)$ & -0.416 & 0.047 \\
\hline Mean arterial blood pressure & & & $0.405(0.344)$ & 0.193 & 0.249 \\
\hline Model 5 & 0.344 & 0.007 & & & \\
\hline Capillary pressure & & & $2.758(1.11)$ & 0.499 & 0.019 \\
\hline BMI & & & $-0.999(0.555)$ & -0.342 & 0.083 \\
\hline Intraocular pressure & & & $1.867(1.012)$ & 0.303 & 0.076 \\
\hline
\end{tabular}

standardised beta $0.568, p=0.008)$, inflammation (Creactive protein; capillary pressure standardised beta $0.544, p=0.011$ ), insulin sensitivity (ITT, capillary pressure standardised beta $-0.483, p \leq 0.022$; HOMA, capillary pressure standardised beta $-0.489, p \leq 0.038$ ), glycaemic status $\left(\mathrm{HbA}_{1 \mathrm{c}}\right.$ and fasting glucose) (capillary pressure

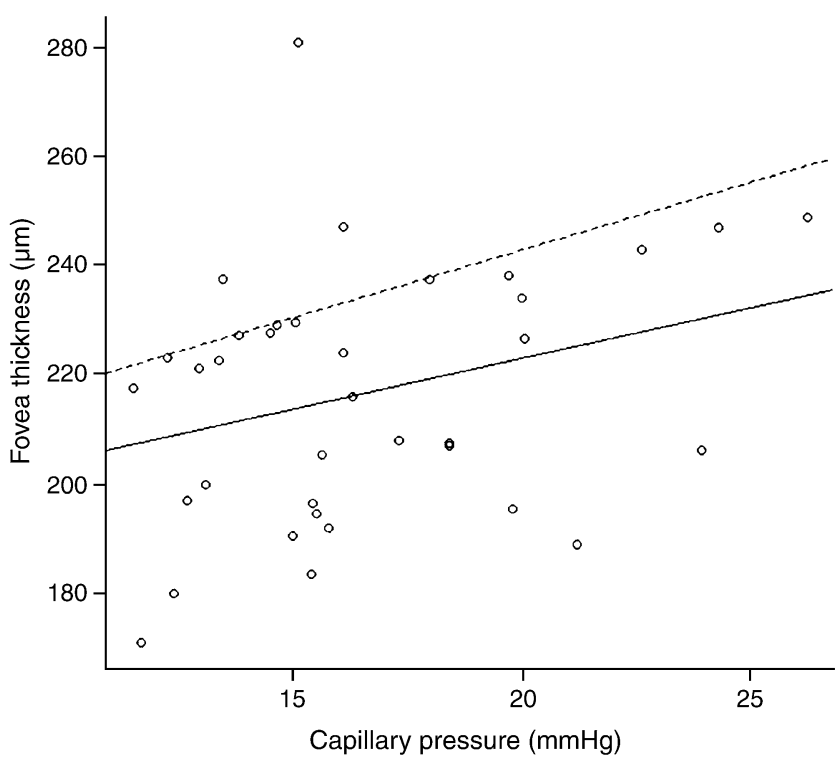

Fig. 1 Association between capillary pressure and fovea thickness. Each marker represents an individual participant. Continuous line, regression line between capillary pressure and fovea thickness $(r=0.379$, $p=0.027)$; dashed line, regression line between capillary pressure and fovea thickness when adjusted for BMI $(r=0.463, p=0.021)$ standardised beta $\geq 0.545, p \leq 0.030$ ) or lipids (capillary pressure standardised beta $\geq 0.513, p \leq 0.019$ ).

\section{Discussion}

This study has demonstrated for the first time that capillary pressure, the key determinant of movement of fluid across a blood vessel wall, is positively associated with fovea thickness in non-diabetic individuals. None of the other microvascular determinants of fluid filtration such as Kf were associated with fovea thickness. In addition, the inner and outer quadrants of the macular were not significantly associated with any of the microvascular variables.

Alterations in fovea thickness (an early sign of macular oedema) are likely to result from changes in transmural capillary fluid movement, which is governed by the balance of hydrostatic and oncotic forces acting across the capillary wall, as described in Starling's equation [30]. Capillary pressure remained associated with fovea thickness when adjusted for intraocular pressure as well as for BMI. Interestingly, the addition of intraocular pressure to the model increased the amount of variance explained by the model from $22 \%$ to $34 \%$. Our observation that a raised hydrostatic capillary pressure capable of promoting fluid filtration is associated with an increase in fovea thickness suggests that alterations in capillary pressure may play an instrumental role in the development of macular oedema. This would be in keeping with the importance previously ascribed to capillary pressure in the movement of fluid across 
the healthy vessel wall, as capillary pressure is known to be the most variable of Starling's forces. These observations suggest that when it comes to potential preventative or therapeutic targets, attention should be directed at the mechanisms determining retinal microvascular pressure.

Microvascular filtration capacity, a component of Starling's equation governing fluid filtration, was not associated with fovea thickness and also did not contribute to the relationship between capillary pressure and fovea thickness. Fovea thickness is thus independent of changes in the surface area available for exchange and permeability of the vessel wall (hydraulic conductance), which determine Kf in non-diabetic, healthy individuals. This observation that structural changes in the microvasculature are not associated with fovea thickness, either independently or in conjunction with capillary pressure, is supported by the lack of relationship of vasodilatory capacity, MVR and microvascular distensibility with fovea thickness in this study. Resting skin perfusion and endothelial function were not associated with fovea thickness. In addition, they did not account for the effect of capillary pressure on fovea thickness, suggesting that this relationship is independent of microvascular skin perfusion.

Acute alterations in fovea thickness have been reported in connection with posture in type 2 diabetes patients with clinically significant macular oedema (CSMO), with the fovea being thicker in the morning and decreasing in thickness during the day and evening [31, 32]. Postureinduced changes in hydrostatic pressure have been proposed to account for these alterations. This is supported by the observation that fovea thickness decreased by a greater magnitude in individuals in the sitting position than in those in the supine position over the course of the day [33]. Changes in venous and capillary pressure are believed to be the main contributors to posture-induced alterations in fovea thickness in type 2 diabetic patients with CSMO [32, 33]. The data in the present study support the importance of capillary pressure in regulation of fovea thickness as was hypothesised in these studies of postural aspects.

Systemic blood pressure did not contribute to the relationship between capillary pressure and fovea thickness, as the relationship remained when controlling for mean, systolic and diastolic arterial blood pressure. This finding is not unexpected as in healthy individuals capillary pressure is independent of arterial blood pressure due to the autoregulation that protects the microcirculation from acute upstream changes in pressure [34]. In contrast, the posture-induced changes to fovea thickness in type 2 diabetic individuals with CSMO have been shown to be independent and dependent of systemic arterial blood pressure [32, 33]. A possible explanation for the potential contribution of arterial blood pressure to posture-induced changes in fovea thickness is a failure of autoregulation in type 2 diabetic patients with retinopathy and macular oedema [35].
Body composition, as reflected by BMI, influenced the relationship between capillary pressure and fovea thickness, as capillary pressure explained more of the variance in fovea thickness when controlling for BMI. This suggests that BMI may mask the effects of capillary pressure on fovea thickness. In fact, there was a consistent trend for increased BMI to be associated with a reduction in fovea thickness. A potential explanation of the negative relationship between BMI and fovea thickness is neurodegeneration, which is associated with obesity [36]. Fovea thickness is reduced in individuals with multiple sclerosis [37]. It is also negatively related to duration of diabetes in type 2 diabetic patients with no or mild retinopathy, a finding thought to reflect neurodegeneration in the diabetic retina [38].

A limitation of the current study is the potential for a type 2 error. However, the fact that capillary pressure was consistently associated with fovea thickness in all models endorses the proposal that an increase in capillary pressure is associated with an increase in fovea thickness.

In summary, this study has shown for the first time that capillary pressure, the key determinant of fluid filtration across the blood vessel, is associated with fovea thickness in non-diabetic individuals. This relationship is independent of arterial and intraocular pressure, BMI, microvascular skin perfusion, Kf, structural changes in the microvasculature, inflammation, glycaemic status, insulin sensitivity and lipids. Thus, our findings support the concept that preclinical changes in microvascular function may be linked with endorgan damage, in this case fovea thickness. They also suggest that in terms of potential preventative or therapeutic targets, attention should be directed at the mechanisms determining retinal microvascular pressure.

Acknowledgements This study was funded by Diabetes UK and supported by the Peninsula NIHR Clinical Research Facility at the University of Exeter. The opinions given in this paper do not necessarily represent those of the NIHR, the NHS or the Department of Health. We are grateful to R. MacLeod for undertaking the retinal photographs and to D. Bedford for her administrative support.

Duality of interest The authors declare that there is no duality of interest associated with this manuscript.

Open Access This article is distributed under the terms of the Creative Commons Attribution Noncommercial License which permits any noncommercial use, distribution, and reproduction in any medium, provided the original author(s) and source are credited.

\section{References}

1. Ikram MK, Janssen JA, Roos AM et al (2006) Retinal vessel diameters and risk of impaired fasting glucose or diabetes: the Rotterdam study. Diabetes 55:506-510

2. Klein R, Klein BE, Moss SE, Wong TY (2006) The relationship of retinopathy in persons without diabetes to the 15 -year incidence 
of diabetes and hypertension: Beaver Dam Eye Study. Trans Am Ophthalmol Soc 104:98-107

3. Nguyen TT, Wang JJ, Islam FM et al (2008) Retinal arteriolar narrowing predicts incidence of diabetes: the Australian Diabetes, Obesity and Lifestyle (AusDiab) Study. Diabetes 57:536-539

4. Wong TY, Shankar A, Klein R, Klein BE, Hubbard LD (2005) Retinal arteriolar narrowing, hypertension, and subsequent risk of diabetes mellitus. Arch Intern Med 165:1060-1065

5. Wong TY, Klein R, Sharrett AR et al (2002) Retinal arteriolar narrowing and risk of diabetes mellitus in middle-aged persons. JAMA 287:2528-2533

6. Kifley A, Wang JJ, Cugati S, Wong TY, Mitchell P (2008) Retinal vascular caliber and the long-term risk of diabetes and impaired fasting glucose: the Blue Mountains Eye Study. Microcirculation 15:373-377

7. Mitchell P, Wang JJ, Wong TY, Smith W, Klein R, Leeder SR (2005) Retinal microvascular signs and risk of stroke and stroke mortality. Neurology 65:1005-1009

8. Wang JJ, Liew G, Klein R et al (2007) Retinal vessel diameter and cardiovascular mortality: pooled data analysis from two older populations. Eur Heart J 28:1984-1992

9. Witt N, Wong TY, Hughes AD et al (2006) Abnormalities of retinal microvascular structure and risk of mortality from ischemic heart disease and stroke. Hypertension 47:975-981

10. Wong TY, Klein R, Couper DJ et al (2001) Retinal microvascular abnormalities and incident stroke: the atherosclerosis risk in communities study. Lancet 358:1134-1140

11. Wong TY, Klein R, Sharrett AR et al (2003) The prevalence and risk factors of retinal microvascular abnormalities in older persons: The Cardiovascular Health Study. Ophthalmology 110:658-666

12. Cooper LS, Wong TY, Klein R et al (2006) Retinal microvascular abnormalities and MRI-defined subclinical cerebral infarction: the Atherosclerosis Risk in Communities Study. Stroke 37:82-86

13. Tikellis G, Arnett DK, Skelton TN et al (2008) Retinal arteriolar narrowing and left ventricular hypertrophy in African Americans. The Atherosclerosis Risk in Communities (ARIC) study. Am J Hypertens 21:352-359

14. Wong TY, Klein R, Nieto FJ et al (2003) Retinal microvascular abnormalities and 10-year cardiovascular mortality: a populationbased case-control study. Ophthalmology 110:933-940

15. Chan NN, Colhoun HM, Vallance P (2001) Cardiovascular risk factors as determinants of endothelium-dependent and endothelium-independent vascular reactivity in the general population. J Am Coll Cardiol 38:1814-1820

16. Ijzerman RG, de Jongh RT, Beijk MAM et al (2003) Individuals at increased coronary heart disease risk are characterized by an impaired microvascular function in skin. Eur J Clin Investig 33:536-542

17. Strain WD, Chaturvedi N, Leggetter $S$ et al (2005) Ethnic differences in skin microvascular function and their relation to cardiac target-organ damage. J Hypertens 23:133-140

18. Strain WD, Chaturvedi N, Bulpitt CJ, Rakijumar C, Shore AC (2005) Albumin excretion rate and cardiovascular risk: could the association be explained by early microvascular dysfunction? Diabetes 54:1816-1822

19. Alberti KGMM, Zimmet P, Shaw J (2006) Metabolic syndromea new world-wide definition. A consensus statement from the International Diabetes Federation. Diabet Med 23:469-480

20. Paunescu LA, Schuman JS, Price LL et al (2004) Reproducibility of nerve fiber thickness, macular thickness, and optic nerve head measurements using StratusOCT. Invest Ophthalmol Vis Sci 45:1716-1724
21. Shore AC, Sandeman DD, Tooke JE (1995) Capillary pressure, pulse pressure amplitude, and pressure waveform in healthy volunteers. Am J Physiol 268:H147-H154

22. Gamble J, Gartside IB, Christ F (1993) A reassessment of mercury in silastic strain gauge plethysmography for microvascular permeability assessment in man. J Physiol 464:407-422

23. Gooding KM, Hannemann MM, Tooke JE, Clough GF, Shore AC (2006) Maximum skin hyperaemia induced by local heating: possible mechanisms. J Vasc Res 43:270-277

24. Morris SJ, Shore AC, Tooke JE (1995) Responses of the skin microcirculation to acetylcholine and sodium nitroprusside in patients with NIDDM. Diabetologia 38:1337-1344

25. Matthews DR, Hosker JP, Rudenski AS et al (1985) Homeostasis model assessment: insulin resistance and $\beta$-cell function from fasting plasma glucose and insulin concentrations in man. Diabetologia 28:412-419

26. Sanchez-Tocino H, Alvarez-Vidal A, Maldonado MJ, MorenoMontanes J, Garcia-Layana A (2002) Retinal thickness study with optical coherence tomography in patients with diabetes. Invest Ophthalmol Vis Sci 43:1588-1594

27. Clough GF, Turzyniecka M, Walter L et al (2009) Muscle microvascular dysfunction in central obesity is related to muscle insulin insensitivity but is not reversed by high-dose statin treatment. Diabetes 58:1185-1191

28. Jonk AM, Houben AJ, de Jongh RT, Serne EH, Schaper NC, Stehouwer CD (2007) Microvascular dysfunction in obesity: a potential mechanism in the pathogenesis of obesity-associated insulin resistance and hypertension. Physiology (Bethesda, MD) $22: 252-260$

29. Chan A, Duker JS, Ko TH, Fujimoto JG, Schuman JS (2006) Normal macular thickness measurements in healthy eyes using Stratus optical coherence tomography. Arch Ophthalmol 124:193-198

30. Michel CC (1997) Starling: the formulation of his hypothesis of microvascular fluid exchange and its significance after 100 years. Exp Physiol 82:1-30

31. Frank RN, Schulz L, Abe K, Iezzi R (2004) Temporal variation in diabetic macular edema measured by optical coherence tomography. Ophthalmology 111:211-217

32. Larsen M, Wang M, Sander B (2005) Overnight thickness variation in diabetic macular edema. Invest Ophthalmol Vis Sci 46:2313-2316

33. Polito A, Del Borrello M, Polini G, Furlan F, Isola M, Bandello F (2006) Diurnal variation in clinically significant diabetic macular edema measured by the Stratus OCT. Retina 26:14-20

34. Shore AC, Sandeman DD, Tooke JE (1993) Effect of an increase in systemic blood pressure on nailfold capillary pressure in humans. Am J Physiol 265:H820-H823

35. Frederiksen CA, Jeppesen P, Knudsen ST, Poulsen PL, Mogensen CE, Bek T (2006) The blood pressure-induced diameter response of retinal arterioles decreases with increasing diabetic maculopathy. Graefes Arch Clin Exp Ophthalmol 244:1255-1261

36. Bruce-Keller AJ, Keller JN, Morrison CD (2009) Obesity and vulnerability of the CNS. Biochim Biophys Acta 1792:395-400

37. Gugleta K, Mehling M, Kochkorov A et al (2008) Pattern of macular thickness changes measured by ocular coherence tomography in patients with multiple sclerosis. Klin Monatsbl Augenheilkd 225:408-412

38. Asefzadeh B, Fisch BM, Parenteau CE, Cavallerano AA (2008) Macular thickness and systemic markers for diabetes in individuals with no or mild diabetic retinopathy. Clin Exp Ophthalmol $36: 455-463$ 